\title{
Combined pulmonary fibrosis and emphysema: bad and ugly all the same?
}

\author{
Vincent Cottin (10)
}

Affiliation: Hospices civils de Lyon, Groupe Hospitalier Est, Service de pneumologie - centre de référence des maladies pulmonaires rares, Université de Lyon, Université Claude Bernard Lyon I, Lyon, France.

Correspondence: Vincent Cottin, National Reference Center for Rare Pulmonary Diseases, Dept of Respiratory Medicine, Groupe Hospitalier Est, Batiment A4 pneumologie, F-69677 Lyon Cedex, France. E-mail: vincent.cottinachu-lyon.fr

@ERSpublications

Patients with CPFE have the same prognosis as those with IPF, despite having generally less extensive fibrosis http://ow.ly/hxSe30c43um

Cite this article as: Cottin V. Combined pulmonary fibrosis and emphysema: bad and ugly all the same? Eur Respir J 2017; 50: 1700846 [https://doi.org/10.1183/13993003.00846-2017].

Emphysema is highly prevalent in patients with idiopathic pulmonary fibrosis (IPF) [1] and interstitial lung disease (ILD) associated with rheumatoid arthritis [2], conditions linked to tobacco smoking [3]. The combination of pulmonary fibrosis and emphysema (CPFE) has been suggested to be a syndrome $[4,5]$, based on distinctive clinical, radiological, functional and outcome features [6]. Indeed, patients with CPFE reproducibly present with a distinct functional profile consisting of relative preservation of lung volumes and greater reduction in diffusing capacity of the lung for carbon monoxide (DLCO) [4]. In a pioneering study, Wells et al. [7] showed that patients with IPF and emphysema had higher lung volumes by $5-10 \%$ and lower DLCO by $\sim 15 \%$ as compared to subjects with IPF alone, after adjustment for the extent of ILD.

Individualisation of CPFE as a syndrome certainly has clinical utility [8], including regarding diagnosis, monitoring of disease progression, clinical research and risk of complications. For example, identifying honeycombing on chest imaging is challenging, as reticulation admixed with emphysema may mimic honeycombing $[9,10]$. Serial trends in forced vital capacity, used for the monitoring of IPF progression, are less reliable in CPFE [11, 12], which has major implications for the routine monitoring of IPF and for trial design [5]. Up to half of the patients with CPFE develop precapillary pulmonary hypertension, with a dismal prognosis [6], and many also die from lung cancer [13].

There remains uncertainty, however, as to whether CPFE represents a distinct entity, especially because a pathogeny unique to CPFE (further to the risk factor of tobacco smoking shared by both components) has not yet been described [8] and because of the heterogeneity of imaging features (figure 1) [14, 15]. There is accumulating evidence that emphysema and fibrosis share a number of molecular and cellular pathways [16], and that genetic traits including short telomeres may predispose smokers to develop both emphysema and fibrosis $[17,18]$. Furthermore, some features seem characteristic of the syndrome and are not observed in either IPF or emphysema alone, especially thick-walled large cysts $[15,19,20]$. However, this is not sufficient to establish that the CPFE syndrome is a distinct entity, and the coincidental clustering of emphysema and fibrosis (two smoking-related conditions) still cannot be excluded [8].

Conceptually, one possible approach to address whether the CPFE syndrome may be a distinct condition would be to demonstrate that its outcome differs from that of IPF alone. However, comparing the outcome of CPFE to that of IPF has revealed very challenging, for a number of reasons. Some series of CPFE have included only patients with IPF and associated emphysema, whereas others have more broadly included other fibrotic ILDs with a potentially better prognosis (fibrotic nonspecific interstitial

Received: April 232017 | Accepted: April 242017

Conflict of interest: Disclosures can be found alongside this article at erj.ersjournals.com

Copyright @ERS 2017 

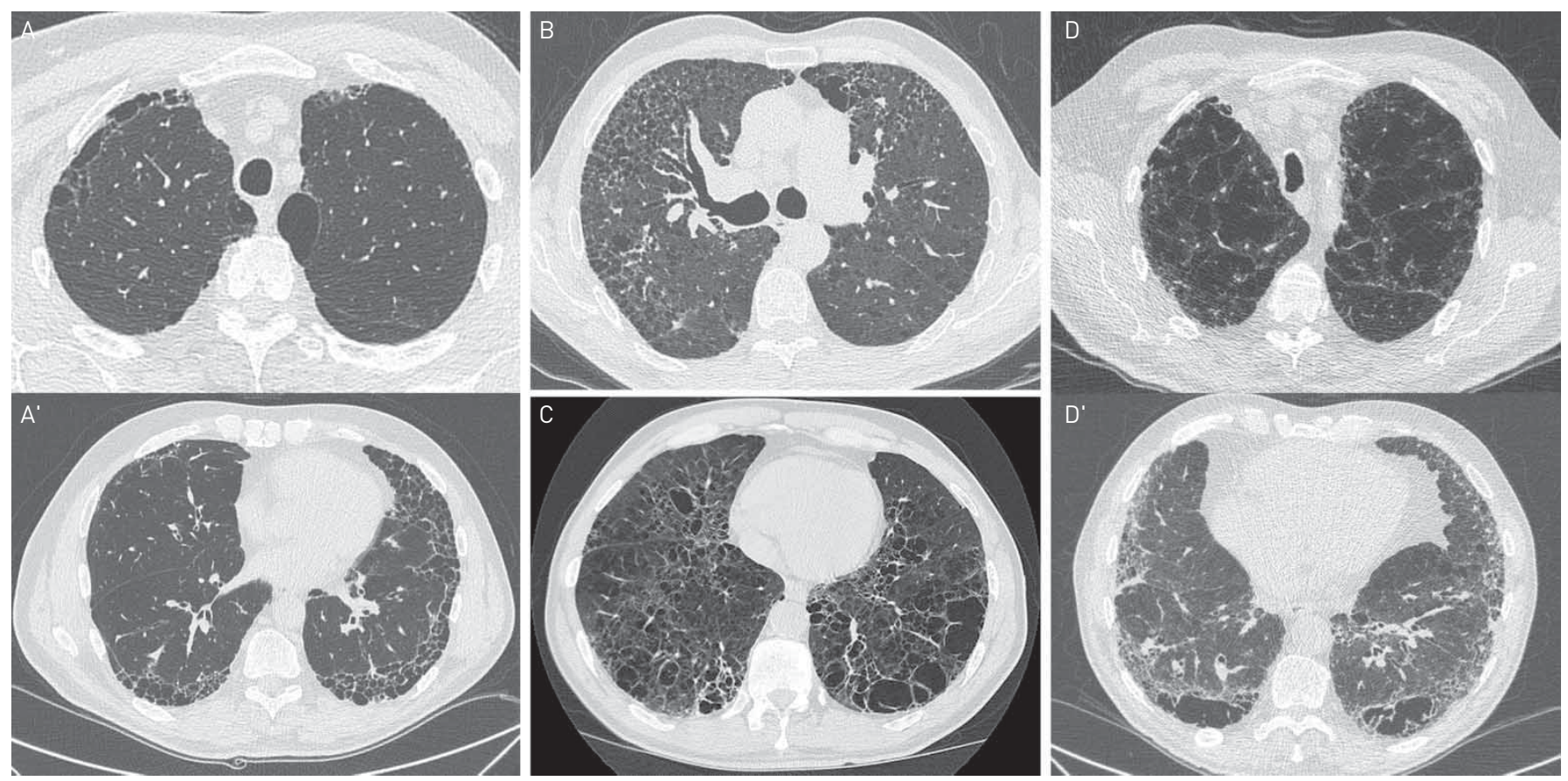

FIGURE 1 Variety of emphysema patterns on high-resolution computed tomography of the chest in patients with combined pulmonary fibrosis and emphysema syndrome. A: paraseptal emphysema in the upper lung zones, distant to fibrosis ("isolated" emphysema); $A^{\prime}$ ': honeycombing in the lower zones in the same patient. B: "admixed" emphysema and fibrosis; cysts are distant from the pleura and have a visible wall, but are not clustered and differ from honeycombing. C: "admixed" emphysema and fibrosis, with diffuse emphysema lesions and thickened walls, with thick-walled large cysts in the left lower lobe, suggesting coexistence of emphysema and fibrosis in the same areas. D: centrilobular emphysema in the upper lung zones; D': subpleural reticulation and thick-walled large cysts in the same patient.

pneumonia, desquamative interstitial pneumonia, unclassifiable pulmonary fibrosis). With some exceptions, most studies have not quantified the extent of fibrosis, and therefore have compared groups of patients with various disease severities at baseline. The calculated rate of functional decline in IPF and CPFE may also have been affected by attrition bias, i.e. calculation of the mean changes in function parameters only in subjects who had survived [11,21], with under-representation at follow-up of patients with the most rapidly progressive disease. As a consequence of these methodological limitations, studies so far have been largely inconclusive and discordant, with different studies suggesting that CPFE may have a worse, similar or even better prognosis than IPF alone. A better appraisal of the prognosis of CPFE and of predictive factors of mortality in this patient population is eagerly needed to prepare for future trials.

To revisit this issue, ЈАСОВ et al. [22] have conducted a very careful evaluation of imaging features in a retrospective cohort of 272 consecutive patients with IPF from their database. They have quantified the extent of emphysema and that of ILD on baseline high-resolution computed tomography (HRCT), using both visual analysis to the nearest 5\% and computer-based analysis with the CALIPER software. Overall, $39 \%$ of the patients had some emphysema on HRCT, and as expected, these had more preserved lung volumes than their counterparts with IPF alone.

The main result of this important study was that the presence or extent of emphysema did not impact survival after correction for baseline severity. The global disease extent on HRCT (total extent of fibrosis and of emphysema) and the baseline DLCO were both predictive of mortality, reflecting the overall severity of parenchymal lung destruction, but the presence of emphysema was not predictive of mortality when adjusting for overall baseline severity.

This important result seems counterintuitive, however, because it means that patients with CPFE would experience the same risk of mortality as those with fibrosis alone, despite their having an additional disease (emphysema). But in multivariate analysis, emphysema was independently predictive of mortality when adjusting for baseline ILD extent (instead of adjusting for baseline DLCO). Interestingly, emphysema extent negatively correlated with the extent of ILD, reproducing earlier studies [14], possibly because patients seek medical advice at a point in time when pulmonary reserve is impaired and the severity of lung involvement generates symptoms, be they related to fibrosis, emphysema or a combination of both. As previously reported [7, 23], patients with CPFE generally had less extensive fibrosis at diagnosis than those with IPF. In other words, patients with CPFE had a similarly poor outcome as those with IPF alone, 
although they generally had a lesser extent of fibrosis, and patients with CPFE had a worse outcome than those with IPF alone for a similar extent of ILD.

IPF did not seem to progress at a different pace when emphysema coexisted and the severity of the lung disease did not differ much between subgroups after adjustment for global disease extent [22]. However, caution should be used when interpreting this finding, pending confirmation in other cohorts. In any study, especially a retrospective study, however excellent in quality, results are limited by the data set and the patient population.

Indeed, one notable limitation of the study by ЈАСОВ et al. [22] is the relatively limited extent of emphysema in the study population, and conclusions might have differed in a population with IPF and more severe emphysema. Virtually no patient had an obstructive ventilatory defect and only 11\% (30 out of 272) patients had $>15 \%$ emphysema extent on HRCT (a threshold recently reported in abstract form, above which emphysema impacts change over time in lung volumes). This low proportion may, at least in part, explain that involvement of the lung parenchyma in emphysema or fibrosis had an apparently comparable impact on overall mortality, which somewhat contradicts clinicians' experience and the literature showing different rates of disease progression between fibrosis and emphysema. Let us take imaginary cases as an (overly simplistic) example. It is hardly conceivable that patient A with 5\% ILD extent and 25\% emphysema extent, and patient B with 25\% ILD extent and 5\% emphysema (patients like those we occasionally see in the clinic), have conditions of similar progression rate and risk of mortality, even though their global (cumulative) extent of disease is comparable; whereas it is easier to conceive that patient C with $20 \%$ ILD and $0 \%$ emphysema, and patient D with $10 \%$ ILD and $10 \%$ emphysema (similar to patients included in the study), may have somewhat comparable outcomes. As a take-home message, in a population of patients with limited extent of emphysema, those with CPFE have the same prognosis as those with IPF, despite having generally less extensive fibrosis, and their fibrosis might progress at a similar rate as that of subjects with IPF alone, but data are not as robust in subjects with more extensive emphysema.

One major difficulty in studying the prognosis of CPFE lies in how to adjust for disease severity, which dramatically affects the study outcome. ЈАСОВ et al. [22] chose to adjust for the global severity of lung disease, evaluated either by baseline DLCO or by total extent of disease at HRCT (calculated by summing emphysema extent and ILD extent). Such a postulate somewhat implies that functional impairment by emphysema is equivalent to functional impairment by fibrosis, i.e. any kind of parenchymal destruction ends up with similar consequences, whatever the mechanism. With this approach, emphysema did not impact prognosis in IPF beyond the additive effects of both fibrosis and emphysema. This method, however, does not allow evaluation of whether emphysema impacts the outcome for a given extent of fibrosis. Furthermore, adjusting for DLCO as a surrogate of disease severity eliminates further differences between groups regarding the risk of pulmonary hypertension, because DLCO already takes into account pulmonary vasculopathy. As DLCO is lower in subjects with pulmonary hypertension and in those who are at high risk of developing pulmonary hypertension, adjusting for DLCO, by definition, makes it impossible to show a difference in the risk of pulmonary hypertension. In future studies, it would be interesting to evaluate whether, for a similar extent of pulmonary fibrosis (a method used in a previous study by the same group [7]), patients with combined emphysema have an increased risk of developing new-onset pulmonary hypertension.

Of note, quantification of ILD extent using CALIPER was marginally stronger than ILD visual scores at mortality prediction [22], adding to recent studies demonstrating that automated quantitative methods have a potential role to predict prognosis in IPF [24-26]. Conversely, visual scores were clearly superior to CALIPER score for the quantification of emphysema, possibly because automated readings of destructive emphysema were confounded by honeycombing. Automated quantification of HRCT is not yet ready for prime time for patients with CPFE.

Interestingly, the authors also evaluated whether the distribution of emphysema with respect to the location of pulmonary fibrosis determined the functional effects of emphysema, a long-standing question that requires careful qualitative and quantitative evaluation of imaging. They found that isolated emphysema (i.e. distant to fibrosis) was independently associated with lower DLCO and transfer coefficient but had no impact on forced vital capacity [22]. In contrast, emphysema admixed within areas of fibrosis was associated with preserved lung volumes, presumably through fibrosis maintaining the distal airways open and preventing their collapse. Therefore, distinct emphysema phenotypes (figure 1) have different impacts on lung function in CPFE, with opposing effects of emphysema on pulmonary volumes and gas transfer.

In conclusion, the study by ЈАСОВ et al. [22] dramatically improves our understanding of the prognosis of patients with smoking-related CPFE syndrome and of its determinants. Importantly, pulmonary fibrosis 
may progress at similar rates in patients with IPF and those with IPF-emphysema (CPFE), and is associated with a high risk of mortality. One relevant consequence for the clinician is that patients with IPF and associated emphysema need treatment, especially when disease behaviour corresponds to progressive fibrosis. Progress made in this area further underlines that an international, consensus definition is needed for CPFE, as a prerequisite to further studies and treatment trials.

\section{References}

1 Mejía M, Carrillo G, Rojas-Serrano J, et al. Idiopathic pulmonary fibrosis and emphysema: decreased survival associated with severe pulmonary arterial hypertension. Chest 2009; 136: 10-15.

2 Antoniou KM, Walsh SL, Hansell DM, et al. Smoking-related emphysema is associated with idiopathic pulmonary fibrosis and rheumatoid lung. Respirology 2013; 18: 1191-1196.

3 Cordier JF, Cottin V. Neglected evidence in idiopathic pulmonary fibrosis: from history to earlier diagnosis. Eur Respir J 2013; 42: 916-923.

4 Cottin V, Nunes H, Brillet PY, et al. Combined pulmonary fibrosis and emphysema: a distinct underrecognised entity. Eur Respir J 2005; 26: 586-593.

5 Cottin V, Cordier JF. The syndrome of combined pulmonary fibrosis and emphysema. Chest 2009; 136: 1-2.

6 Cottin V, Le Pavec J, Prévot G, et al. Pulmonary hypertension in patients with combined pulmonary fibrosis and emphysema syndrome. Eur Respir J 2010; 35: 105-111.

7 Wells AU, King AD, Rubens MB, et al. Lone cryptogenic fibrosing alveolitis: a functional-morphologic correlation based on extent of disease on thin-section computed tomography. Am J Respir Crit Care Med 1997; 155: $1367-1375$.

8 Wells AU, Margaritopoulos GA, Antoniou KM, et al. CPFE: distinctive and non-distinctive features. In: Costabel U, Crestani B, Wells AU, et al., eds. Idiopathic Pulmonary Fibrosis (ERS Monograph). Sheffield, European Respiratory Society, 2016; pp. 175-185.

9 Akira M, Inoue Y, Kitaichi M, et al. Usual interstitial pneumonia and nonspecific interstitial pneumonia with and without concurrent emphysema: thin-section CT findings. Radiology 2009; 251: 271-279.

10 Watadani T, Sakai F, Johkoh T, et al. Interobserver variability in the CT assessment of honeycombing in the lungs. Radiology 2013; 266: 936-944.

11 Akagi T, Matsumoto T, Harada T, et al. Coexistent emphysema delays the decrease of vital capacity in idiopathic pulmonary fibrosis. Respir Med 2009; 103: 1209-1215.

12 Schmidt SL, Nambiar AM, Tayob N, et al. Pulmonary function measures predict mortality differently in IPF versus combined pulmonary fibrosis and emphysema. Eur Respir J 2011; 38: 176-183.

13 Girard N, Marchand Adam S, Naccache JM, et al. Lung cancer in combined pulmonary fibrosis and emphysema: a series of 47 Western patients. J Thorac Oncol 2014; 9: 1162-1170.

14 Brillet $\mathrm{P}$, Cottin V, Letoumelin $\mathrm{P}$, et al. Syndrome emphysème des sommets et fibrose pulmonaire des bases combinés (syndrome emphysème/fibrose): aspects tomodensitométriques et fonctionnels [Combined apical emphysema and basal fibrosis syndrome (emphysema/fibrosis syndrome): CT imaging features and pulmonary function tests]. J Radiol 2009; 90: 43-51.

15 Cottin V, Cordier JF. The syndrome of combined pulmonary fibrosis and emphysema. In: Cottin V, Cordier JF, Richeldi L, eds. Orphan Lung Disease: A Clinical Guide to Rare Lung Disease. London, Springer Verlag, 2015; pp. 327-347.

16 Kusko RL, Brothers JF 2nd, Tedrow J, et al. Integrated genomics reveals convergent transcriptomic networks underlying chronic obstructive pulmonary disease and idiopathic pulmonary fibrosis. Am J Respir Crit Care Med 2016; 194: 948-960.

17 Cottin V, Reix P, Khouatra C, et al. Combined pulmonary fibrosis and emphysema syndrome associated with familial SFTPC mutation. Thorax 2011; 66: 918-919.

18 Nunes H, Monnet I, Kannengiesser C, et al. Is telomeropathy the explanation for combined pulmonary fibrosis and emphysema syndrome? Report of a family with TERT mutation. Am J Respir Crit Care Med 2014; 189: 753-754.

19 Inomata M, Ikushima S, Awano N, et al. An autopsy study of combined pulmonary fibrosis and emphysema: correlations among clinical, radiological, and pathological features. BMC Pulm Med 2014; 14: 104.

20 Cottin V, Nunes H, Mouthon L, et al. Combined pulmonary fibrosis and emphysema syndrome in connective tissue disease. Arthritis Rheum 2011; 63: 295-304

21 Kim YJ, Shin SH, Park JW, et al. Annual change in pulmonary function and clinical characteristics of combined pulmonary fibrosis and emphysema and idiopathic pulmonary fibrosis: over a 3-year follow-up. Tuberc Respir Dis 2014; 77: 18-23.

22 Jacob J, Bartholmai BJ, Rajagopalan S, et al. Functional and prognostic effects when emphysema complicates idiopathic pulmonary fibrosis. Eur Respir J 2017; 50: 1700379.

23 Ryerson CJ, Hartman T, Elicker BM, et al. Clinical features and outcomes in combined pulmonary fibrosis and emphysema in idiopathic pulmonary fibrosis. Chest 2013; 144: 234-240.

24 Maldonado F, Moua T, Rajagopalan S, et al. Automated quantification of radiological patterns predicts survival in idiopathic pulmonary fibrosis. Eur Respir J 2014; 43: 204-212.

25 Salisbury ML, Lynch DA, van Beek EJ, et al. Idiopathic Pulmonary Fibrosis: The Association between the Adaptive Multiple Features Method and Fibrosis Outcomes. Am J Respir Crit Care Med 2017; 195: 921-929.

26 Jacob J, Bartholmai BJ, Rajagopalan S, et al. Mortality prediction in idiopathic pulmonary fibrosis: evaluation of computer-based CT analysis with conventional severity measures. Eur Respir J 2017; 49: 1601011. 